\title{
Civilisations
}

Revue internationale d'anthropologie et de sciences

humaines

66 | 2017

L'alcool rituel et les ethnographes

\section{La part des morts. Les usages de l'alcool dans le culte du palo monte afro-cubain}

\section{Katerina Kerestetzi}

\section{OpenEdition}

\section{Journals}

Édition électronique

URL : http://journals.openedition.org/civilisations/4181

DOI : 10.4000/civilisations.4181

ISSN : 2032-0442

\section{Éditeur}

Institut de sociologie de l'Université Libre de Bruxelles

\section{Édition imprimée}

Date de publication : 31 août 2017

Pagination : 25-41

ISBN : 978-2-9602017-1-0

ISSN : 0009-8140

\section{Référence électronique}

Katerina Kerestetzi, «La part des morts. Les usages de l'alcool dans le culte du palo monte afrocubain », Civilisations [En ligne], 66 | 2017, mis en ligne le 31 août 2020, consulté le 25 février 2021. URL : http://journals.openedition.org/civilisations/4181; DOI : https://doi.org/10.4000/civilisations. 4181 


\title{
La part des morts
}

\section{Les usages de l'alcool dans le culte du palo monte afro-cubain}

\author{
Katerina KERESTETZI
}

Résumé : Dans la pratique du culte initiatique afro-cubain du palo monte, l'alcool est omniprésent. Rhum, eau-de-vie, bière, alcools purs coulent à flots lors des rituels, des consultations, des nettoyages rituels et des divinations; ils sont bus, versés, crachés, soufflés, enflammés, enduits. Ils servent à purifier les adeptes, à détruire le mal, à contenter les esprits... Chacun des usages de l'alcool est fortement encadré et codifié, requiert de maîtriser des techniques et des gestes et présuppose l'intériorisation de certains schèmes interprétatifs sur son efficacité. Par ailleurs, la manière d'employer les spiritueux varie considérablement d'un groupe initiatique à l'autre. Certains encouragent une grande consommation de rhum, ce qui rend les cérémonies particulièrement festives ou conviviales, d'autres en font un usage très modéré afin de maintenir les participants dans un état de concentration optimale. Chez d'autres encore, l'alcool sert de levier performatif et on y voit les médiums engloutir des bouteilles entières de rhum parce que les morts qui prennent possession de leur corps sont « alcooliques ».

Mots-clés : palo monte, religions afro-cubaines, style rituel, alcool, morts, pragmatique rituelle, affordances.

\begin{abstract}
Alcohol is omnipresent in the practice of the Afro-Cuban initiating religion of Palo Monte. Rum, aguardiente, beer, pure alcohol flow during rituals, consultations, ritual cleansings and divinations; they ares drunk, shed, spat, blown, inflamed, smeared. Alcohol serves to purify the adepts, to destroy the evil, to satisfy the spirits ... Its uses are highly framed and codified, they require the mastering of various techniques and gestures and presuppose the internalization of certain interpretative schemes concerning its effectiveness. In addition, the use of alcohol varies considerably from one initiation group to another. Some encourage a high consumption of rum, which makes the ceremonies particularly festive or convivial, others use it in moderation in order to keep participants in a state of optimal concentration. For others, alcohol is a performative lever and the mediums are seen to swallow whole bottles of rum because the dead who take possession of their bodies are "alcoholic".
\end{abstract}

Keywords: Palo Monte, Afro-Cuban religions, ritual style, alcohol, dead, ritual pragmatics, affordances 


\section{Introduction}

Si l'on pouvait adopter, le temps d'une cérémonie du culte du palo monte afro-cubain, le point de vue d'une bouteille de rhum, on aurait vite le tournis et on serait surpris du peu de répit qu'on lui laisse. Elle est bue, crachée, vaporisée, versée, enflammée... Dans la pratique de ce culte initiatique très répandu à Cuba, les alcools, comme le rhum mais aussi l'eau-de-vie, la bière, les alcools purs et même les parfums, sont omniprésents. Ils coulent à flots lors des rituels, des consultations, des nettoyages rituels et des divinations. Ils servent à détruire le mal, à contenter les esprits, à animer l'ambiance, à purifier les animaux destinés aux sacrifices, à calmer les vibrations meurtrières des couteaux ${ }^{1} \ldots$

Si l'alcool est aussi présent dans les lieux de culte du palo monte, les cuartos de fundamento [littéralement, " pièces de fondation »], c'est parce que là vivent des morts tout-puissants, les nfumbis, qui tous possèdent, à des degrés divers, un certain penchant pour la bouteille. Ce sont eux qui sont au cœur du culte du palo monte, qui sont à la source des pouvoirs de ses initiés et qui sont les destinataires de leurs prières. Pour mettre ces morts à leur service, les pratiquants du palo monte vont prélever secrètement certains de leurs os dans un cimetière puis les installent chez eux, dans de larges chaudrons de terre cuite ou de fer, les ngangas. Ils leur permettent ainsi de retrouver certains attributs propres à la vie : jouissance, chaleur, faim et soif. Les pratiquants du palo monte, les paleros, doivent être attentifs aux besoins des nfumbis, les nourrir et les désaltérer régulièrement (alimentar) (Figures 1,2) et ce principalement avec des offrandes liquides (boissons alcoolisées, café, miel et sang). Parmi elles, le rhum et l'eau-de-vie constituent les éléments principaux de la diète quotidienne des nfumbis. Et pour cause : la plupart d'entre eux ont été alcooliques de leur vivant et leur goût pour l'alcool ne s'est pas arrêté avec leur mort. Les nfumbis se recrutent souvent parmi les personnes décédées de manière subite (accident de la route, assassinat, crise cardiaque) qui n'ont pas eu le temps de réaliser qu'elles quittaient la vie. Elles continuent à se croire vivantes et conservent leurs anciennes habitudes humaines, y compris leurs vices, parmi lesquels figure en bonne place la consommation d'alcool.

Les paleros préfèrent généralement les esprits d'individus ayant vécu en marge de la société : voyous, assassins, ou encore malades mentaux. Non pas parce que les paleros ont une appétence morbide pour les êtres déviants, mais pour la raison pragmatique que de tels esprits n'auront aucun scrupule à effectuer des opérations moralement répréhensibles. On pourra leur demander de séparer des couples, d'engendrer des maladies, et dans des cas extrêmes de tuer même, tout en sachant qu'ils pourront toujours guérir, apporter le réconfort, la richesse, l'amour...

1 Les données présentées dans cet article sont issues d'une longue enquête de terrain à Cuba, dans la région de Cienfuegos, sur le culte du palo monte, qui a commencé en 2003 et qui continue jusqu'aujourd'hui. Le palo monte est organisé en groupes initiatiques indépendants ou « familles de religion », selon le terme des pratiquants, qui sont dirigés par des chefs rituels (hommes et/ou femmes), les «parrains » (padrinos) spirituels des initiés. Il n'y pas d'instance ou d'autorité centralisée pour systématiser la liturgie palera. Ces groupes et leurs chefs ont ainsi la liberté de définir leur pratique religieuse en toute liberté, d'innover et de se distinguer les uns des autres. 


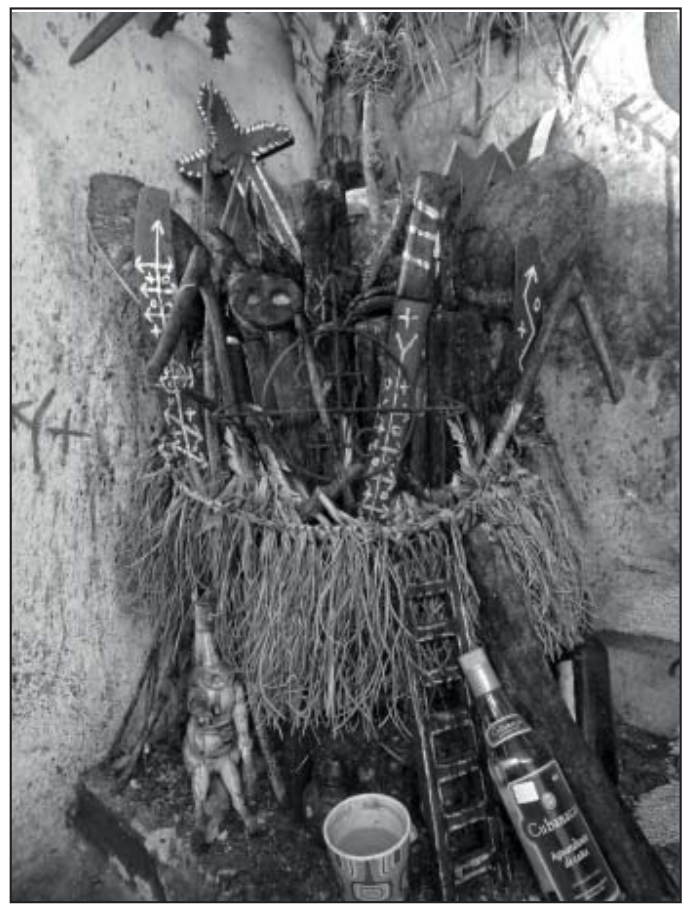

Figure 1 : Une nganga. La bouteille d'eau-de-vie à ses pieds fait presque partie de son esthétique (c) Katerina Kerestetzi, Palmira 2015

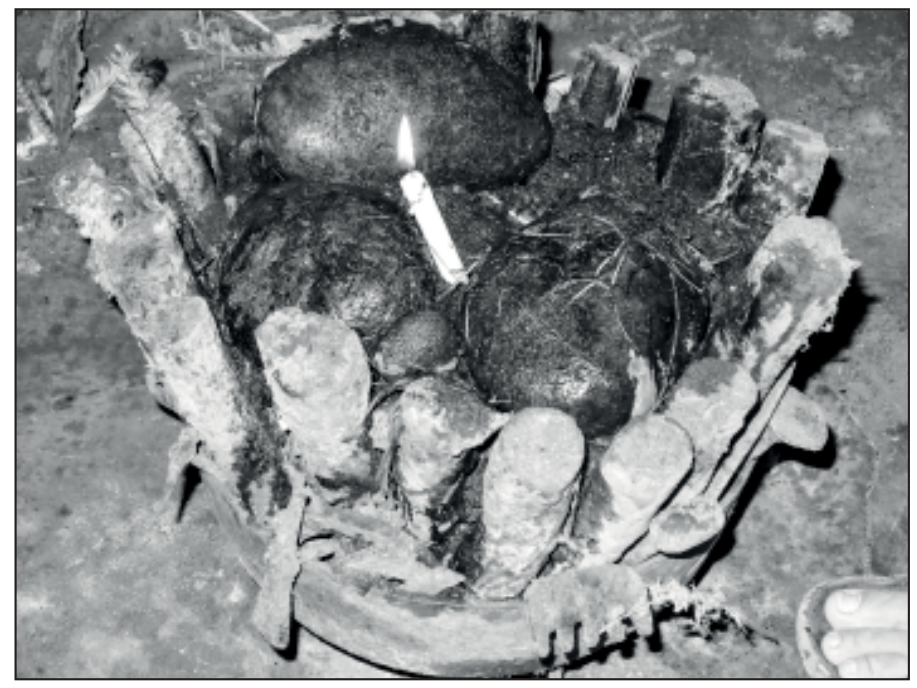

Figure 2 : Nganga aspergée de rhum - (C) Katerina Kerestetzi, Cienfuegos 2012 
Dans le marché spirituel cubain, très concurrentiel ${ }^{2}$, cette polyvalence des nfumbis constitue un indéniable atout praxique car il permet aux paleros d'effectuer tout type d'opération magique, ce qui contribue à élargir la base de leur clientèle qui monnaie leurs services. Comme le disent les pratiquants-mêmes, " avec les nfumbis, nous pouvons tout faire, satisfaire tous les souhaits ». Ceci étant dit, les morts sont aussi pour les pratiquants des compagnons de vie qu'ils aiment comme des frères, mais aussi des êtres divins qu'ils vont consulter pour leur insondable sagesse. Cette ambiguïté - et on parle ici de trois ontologies morales (celle de l'assassin, de l'ami bienveillant et de l'être divin) -, le fait qu'ils sont présentés à la fois comme maléfiques et comme profondément bons et aimables est au fondement de la fascination qu'ils suscitent. La tension entre les deux facettes de leur personnalité, instillant tant de peur que d'émerveillement, se converti ainsi en émotion religieuse. La propension éthylique des nfumbis participe de cette dualité puisque la consommation d'alcool est elle-même ambigüe d'un point de vue moral, s'agissant à la fois d'une activité favorisant les conduites dissolues et excessives mais aussi d'une affaire profondément sociale et conviviale. Rien d'étonnant alors que les discours des paleros eux-mêmes soient si contrastés lorsque l'on aborde la question de l'alcool dans le palo monte : tantôt il s'agit d'un précieux instrument destiné à « alimenter » et honorer les morts et tantôt il constitue un argument pour discréditer les pratiques de paleros concurrents - les récits d'ivrognes qui offensent leurs morts par des comportements déplacés sont monnaie courante. Ce dernier aspect n'est d'ailleurs pas sans lien avec la mauvaise réputation des paleros à Cuba, considérés comme les plus immoraux des religieux.

Il y a enfin une autre dimension éminemment pratique de l'usage de l'alcool qui ne peut être comprise que si l'on aborde la question de la psyché des nfumbis. Pour les paleros, ces âmes sont issues de personnes qui ont été rejetées ou abandonnées par leur famille de leur vivant. Abandon qui s'est poursuivi dans la mort et qui s'est traduit par une désertion de leur tombe. Or, à Cuba, sous l'influence du spiritisme (espiritismo), dont la théorie sur les âmes des défunts a pénétré tous les cultes afro-cubains (voir Palmié 2002 : 192), il est notoire que l'on peut aider un mort à s'épurer et à s'élever dans le monde des esprits, en déposant des fleurs et des bougies sur sa tombe et en effectuant

2 La religion à Cuba constitue un système riche et complexe : on y assiste au foisonnement des Eglises évangéliques, baptistes, méthodistes, pentecôtistes, des témoins de Jéhovah, du catholicisme, qui se développent avec le même rythme intense que les religions afro-cubaines. Elles incluent : le palo monte, d'influence bantoue ; la santería, d'influence yoruba, dont les pratiquants, les santeros, développent une intimité avec des divinités anthropomorphes ; le culte d'Ifá, corollaire de la santería et d'influence yoruba également, qui recrute uniquement des hommes, les babalaos, se dédiant à la divination ; la société secrète masculine des Abakuás (d'origine efik-ibibio) qui se présente davantage comme un « club de prestige », tout comme la franc-maçonnerie ; et finalement, le espiritismo syncrétique, une variante du spiritisme euro-américain, fondé sur la communication avec les morts. Le espiritismo s'est implanté à Cuba au cours du 19e siècle et qui est pratiqué par les pratiquants des cultes afro-cubains, desquels il a emprunté plusieurs éléments liturgiques. La majorité des adeptes de ces religions est de manière régulière ou occasionnelle impliquée simultanément dans plusieurs de ces cultes ou passe de l'un à l'autre au cours de sa vie. Rien n'empêche a priori un Cubain d'être à la fois palero, santero, spirite et catholique (et communiste). Sur les religions afro-cubaines, voir entre beaucoup d'autres : Ortiz 2001 [1906] ; Cabrera 1998 [1954]; Castellanos et Castellanos 1992 ; Argyriadis 1999 ; Palmié 2002. 
des messes en sa mémoire ${ }^{3}$. En l'absence de ces opérations, les défunts deviennent des esprits «non évolués » (atrasados), " obscurs » (oscuros), " mauvais » (malos) et « matérialistes » (materialistas), c'est-à-dire très attachés au monde matériel, des qualificatifs qui décrivent assez bien les nfumbis du palo monte. Mais à la différence des spirites ou des santeros qui voient dans cet état une dégénérescence ontologique de l'âme du défunt, les paleros, eux, y voient une aubaine : en donnant aux nfumbis l'attention et les biens matériels dont leur famille les a cruellement privés, ils reçoivent en retour loyauté et dévouement à toute épreuve. Ajoutons à cela l'offrande d'une substance addictive comme l'alcool et le tour est joué : le pratiquant aura une emprise inégalée sur son mort, celui-ci lui obéissant afin d'obtenir sa « dose ».

Dans cet article, nous verrons comment l'alcool, un produit de consommation très courante à Cuba, est converti en instrument rituel par la manière très singulière dont les adeptes du palo monte l'utilisent et le conceptualisent. Nous montrerons notamment que le rôle qu'il joue dans la ritualité palera ne repose pas uniquement sur sa capacité à générer l'ivresse mais qu'il peut également être attribué à la très grande diversité de ses affordances. Par « affordance », je fais référence au terme utilisé dans la psychologie de la perception (Gibson 1977) qui désigne la capacité d'un objet à suggérer sa propre utilisation par sa seule constitution matérielle - par exemple, une tasse de café nous invite à la tenir par la poignée. Nous verrons ainsi que l'alcool dans le palo monte invite à des emplois fort hétérogènes qui dépassent largement la sphère du boire : le fait par exemple qu'il est inflammable peut susciter des envies de pyrotechnie au service du spectacle rituel ; sa propriété de désinfectant peut faire de lui un purificateur spirituel, etc. Nous verrons que cette multifonctionnalité génère un vaste répertoire d'actions, de techniques du corps et même de vocations d'artisan qui viennent enrichir l'appareil liturgique du culte. Partant d'une pragmatique de l'usage de l'alcool en contexte rituel, fondée sur une description ethnographique détaillée, il s'agira de définir la manière dont ce véritable outil rituel participe à l'organisation des cérémonies du palo monte et à leur dramaturgie. Nous verrons aussi comment la très grande plasticité de ses usages intervient dans la création de styles rituels idiosyncrasiques.

L'intention ici est de proposer, en quelque sorte, une entrée par la petite porte dans l'univers d'une pratique religieuse et de démontrer la valeur heuristique de l'analyse des multiples expressions d'un élément, en apparence secondaire. Jusqu'à présent, cette « anthropologie de l'anodin » n'a que peu motivé la curiosité des chercheurs du champ d'étude afro-cubain ou plus généralement afro-américain. Sur la question spécifique de l'alcool, on ne peut que constater la quasi-absence des études ${ }^{4}$. De manière générale (et mes travaux n'y échappent pas) la focale est généralement tournée vers les «points chauds du religieux », à savoir les esprits, les dieux, les mythes, l'organisation sociale et liturgique... Pourtant, nul doute qu'il y a fort à apprendre de ces oubliés du rituel, éléments restés dans l'ombre du regard et de l'écriture anthropologique.

3 La théorie du spiritisme syncrétique est très influencée de la doctrine d'Allan Kardec, notamment telle qu'elle est formulée dans son œuvre « Le livre des esprits » (2006 [1857]).

4 Pour des travaux sur les offrandes alimentaires dans les religions afro-américaines, voir Bonvini 1996 ; Rougeon et Santiago 2017. 


\section{Boire avec les morts}

Les pratiquants disent que les morts aiment boire et manger ce que leurs paleros boivent et mangent. On est là face à l'expression d'un esprit de symétrie et d'égalité caractéristique du culte. Le partage et la consommation commune des offrandes avec les êtres spirituels distingue le palo monte d'autres cultes afro-cubains, moins égalitaires, qui la proscrivent : ainsi les divinités légendaires de la santería, les orichas, se trouvant dans une relation de supériorité vis-à-vis des hommes, se vexeraient de savoir leurs offrandes spoliées par ces derniers. Boire de leur rhum signifierait, sinon un sacrilège, au moins une atteinte à la hiérarchie divine. Dans le palo monte, la notion de hiérarchie perd son sens. L'initié n'est pas inférieur à son référent spirituel car il l'a crée et le nourrit et il ne lui est pas supérieur non plus car il est moins sage et moins puissant. Tous deux sont liés par une relation de réciprocité. Boire avec les morts est donc un acte politique, même s'il s'agit ici de politique cosmique.

Boire avec les morts est aussi une routine. Car c'est presque tous les jours que les pratiquants rendent visite à leurs morts pour demander conseil ou pour consulter des clients, pour faire un sort ou une divination. Et chaque visite est assortie d'une libation à la nganga, qu'il s'agisse de verser ou de cracher un peu de rhum ou d'eau-de-vie. Cette proximité du pratiquant et de ses morts est éminemment liée à leur proximité géographique. Car ils habitent, au sens littéral du terme, ensemble; les chaudrons des deuxièmes sont installés au sein de l'espace habité.

La symétrie et la réciprocité entre humains et nfumbis est donnée également à voir par un geste qui s'apparente à une banale politesse. Lorsqu' on se rend dans un sanctuaire palero, chez les morts donc, il est recommandé que l'on n'arrive pas les mains vides - tout comme quand on est invité à une soirée chez des amis. L'offrande la plus courante qu'un initié puisse faire aux morts est une bouteille d'alcool. Mais comme il ne s'agit tout de même pas d'une soirée banale, le type de bouteille offerte n'est pas laissé au hasard ; là encore les discours abondent. Le meilleur alcool qu'on puisse offrir aux morts est l'eau-de-vie (aguardiente en espagnol) que l'on nomme " malafo maputo » selon la terminologie rituelle, inspirée du kikongo (langue bantoue). Issue de la canne à sucre, cette boisson est comme la madeleine de Proust pour les esprits du palo monte car elle évoque les plantations de canne dans lesquelles travaillaient les esclaves originaires d'Afrique qui sont les fondateurs supposés du palo monte - le culte a hérité de plusieurs éléments cultuels bantous, introduits à Cuba avec les esclaves originaires de l'Afrique centrale. De plus, cette eau-de-vie, ne contenant pas de rajouts chimiques, renvoie à la pureté de la Nature, à laquelle les paleros vouent un culte particulier, au point qu'ils définissent le palo comme « un culte de la Nature ». La suprématie de cet alcool sur les autres est telle que dans certains groupes, à défaut d'eau-de-vie, on doit combiner sept autres boissons alcoolisées différentes ${ }^{5}$.

Le rhum, le malafo, est tout aussi populaire pourvu qu'il soit de couleur claire, ou mieux encore, qu'il soit parfaitement transparent. Au critère de la pureté de l'alcool s'ajoute donc un deuxième, celui de sa limpidité. Les pratiquants distinguent en

5 Le chiffre 7 tient une place toute particulière dans la tradition palera et on le retrouve, ainsi que ses multiples, dans la composition de nombreuses recettes magiques. Dans cet exemple, je ne peux préciser la nature exacte de ces 7 boissons. 
effet boissons alcooliques «claires » (bevidas claras) et boissons sombres (bevidas oscuras). Cette classification binaire ne procède pas d'un caprice gustatif des nfumbis mais d'une triste réalité du palo monte, à savoir la peur obsessionnelle des paleros d'être empoisonnés physiquement ou magiquement par l'un de leurs concurrents. Il est d'usage en effet dans le culte de réduire en poudre les ingrédients d'un sortilège puis de les verser discrètement dans un breuvage destiné à un ennemi, généralement de couleur foncée, comme un café par exemple. On peut même ensorceler un nfumbi de la sorte : les histoires de paleros abondent de cas de ngangas dont la puissance a été anéantie à cause du manque de vigilance de leur possesseur. Ainsi, aucune boisson alcoolisée sombre n'est autorisée pour les nfumbi : ni whisky ni anejo (du rhum vieilli) pour eux, à moins que leurs chefs se soient portés garants de leur contenu. Notons que certains pratiquants préfèrent à cette explication peu flatteuse pour la communauté palera, celle plus symbolique qui veut que l'on choisisse des breuvages exclusivement translucides parce que « la clarté de la boisson éclaire les chemins de la nganga ».

Fondamentalement, on peut dire que boire avec les morts est une manière de rendre coprésents les initiés et les morts, dans un même espace, le temps d'un rituel, et d'afficher « une constante réciprocité » (Geffroy 2012 : 87) ${ }^{6}$. Pour le pratiquant du palo monte, il est essentiel de manifester régulièrement ce lien d'intimité avec les morts en public, car c'est lui-seul qui lui procure la légitimité nécessaire. En effet, le palero ne possède pas de pouvoir en propre si ce n'est celui de convaincre son nfumbi d'agir en son nom. Et pour ce faire, compte tenu de la dissymétrie des forces en présence, le palero doit davantage compter sur l'amour que sur la violence pour convaincre cet esprit omnipotent et omniscient de travailler pour lui. Ainsi s'instaure entre les deux une relation affective que certains qualifient d'amicale ou même de familiale ; certains paleros parlent volontiers de leur nfumbi comme d' " un ami, d'un frère, d'un père ». La commensalité éthylique est la manifestation praxique de cette relation intime. Rien d'étonnant alors que les pratiquants en fassent une obligation rituelle à part entière. Pour certains, cela requiert quelques sacrifices. Jorge, par exemple, qui est un vieux palero, suit un régime alimentaire très strict dont l'alcool est formellement exclu car il souffre de problèmes cardiaques. Or, quand il officie, quand il reçoit des clients ou quand il effectue un rituel collectif, il ne peut faire autrement que de boire du rhum car son $n f u m b i$ le lui demande. Mais cela ne lui fait pas peur car selon lui, il ne craint rien : si son mort l'incite à boire, c'est qu'il sait aussi comment protéger sa santé.

\section{L'alcool en tant que levier performatif ${ }^{7}$}

L'usage de l'alcool participe activement à l'organisation des rituels et des consultations. Première règle : les morts boivent en premier et cela vaut aussi bien dans les sanctuaires que dans les lieux les plus triviaux. Un palero qui passe du bon temps avec ses amis à la plage prendra toujours soin de verser quelques gouttes sur le sable ou dans la mer avant de se servir lui-même. Notons que ce simple geste effectué en public permet de témoigner de la prééminence des morts sur les vivants et d'actualiser son

6 Sur les dispositifs de co-présence et de co-activité avec les entités spirituelles, voir aussi Stépanoff 2012 et Pitrou 2012.

7 Par l'expression « levier performatif », je veux dire que la manipulation de l'alcool permet des usages très variés qui donnent du dynamisme à la performance rituelle. 
rapport à la religion en dehors des espaces liturgiques. Dans un sanctuaire, cette règle de la préséance des ngangas sur les humains se double de la préséance des ngangas les unes sur les autres et l'on commence par abreuver la nganga principale, la cheftaine du sanctuaire, avant de distribuer l'alcool aux autres ngangas ${ }^{8}$.

L'alcool est aussi un outil au service du spectacle rituel car il ouvre les portes d'un répertoire praxique et kinésique très varié : boire, verser, cracher, souffler, enflammer, enduire. En se référant à l'alcool, Michael Dietler (2006 : 231) parle d'une culture matérielle somatisée (embodied material culture). Ce terme prend toute sa dimension dans le cas du palo monte avec le mode d'administration de l'alcool aux esprits : l'officiant prend une grande gorgée de rhum et la projette avec puissance sur toute la hauteur de la nganga. L'effet est assez spectaculaire car la pression de l'air exercée sur les lèvres divise le liquide en infimes particules, à la manière d'un brumisateur. Parfois les paleros accompagnent cette action d'une posture corporelle particulière ce qui accroit son effet théâtral (Figure 3). Cette technique est loin d'être facile à reproduire. En dépit de nombreuses tentatives personnelles, je n'ai jamais réussi l'opération : en général la moitié du liquide se répand sur mes vêtements, ce qui ne manque pas de provoquer l'hilarité des spectateurs. Ceci pour dire que cette manière de propulser l'alcool demande un apprentissage à part entière et une conduite motrice si singulière qu'elle mériterait que l'on invente un nouveau mot pour la décrire. Les paleros, quant à eux, la nomment « souffler à la nganga» (soplar a la nganga).

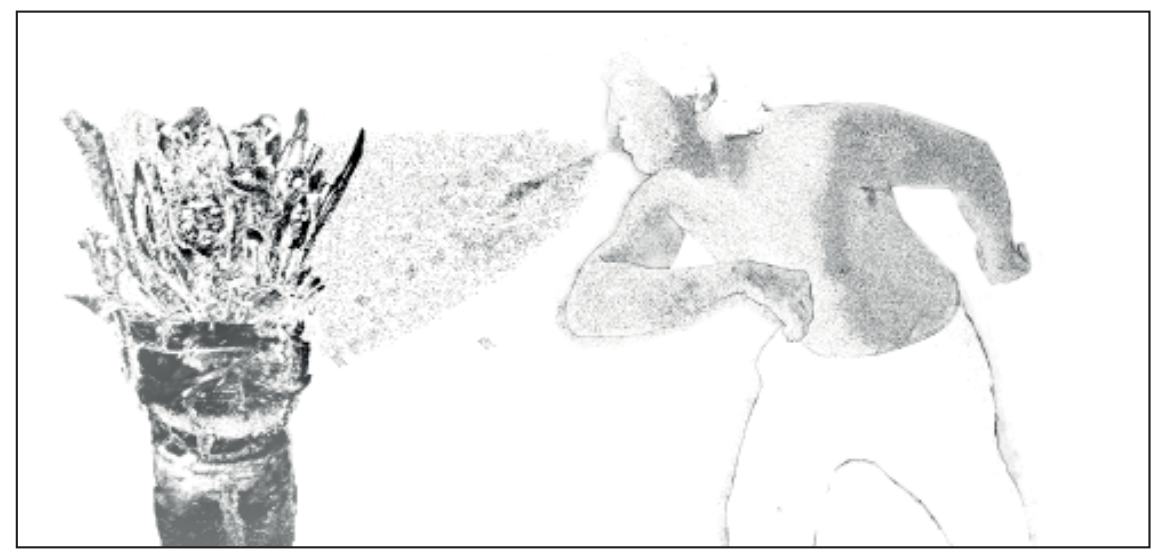

Figure 3 : Palero qui donne à boire à sa ndanga - (c) Katerina Kerestetzi

Une fois les morts désaltérés, on fait circuler le rhum entre les participants qui sont tenus d'en boire une gorgée. Chacun doit tenir le récipient qu'on leur tend, mains croisées et boire de la même façon (Figure 4). Croiser les mains est un moyen d'éviter de « se faire voler son énergie " par un autre, comme le disent les pratiquants, toujours en vertu de cette méfiance qu'ils ont à l'égard de leurs coreligionnaires. Ce geste est d'ailleurs répété à chaque fois que l'on transmet un objet matériel dans un rituel. Dans le cas de l'alcool, il a pour effet de contraindre celui qui boit à amplifier son mouvement de tête vers l'arrière, ce qui accentue l'effet dramaturgique du geste de boire, lui ôtant du même coup une part de sa banalité.

8 Chaque sanctuaire dans le palo monte est privé et la plupart des objets rituels appartiennent au palero qui en est le dépositaire. Chaque palero peut avoir plusieurs ngangas mais il en distingue habituellement une qui fait office de chef et d'organisateur des actions des autres. Le plus souvent, il s'agit de la première nganga que le palero a monté. 


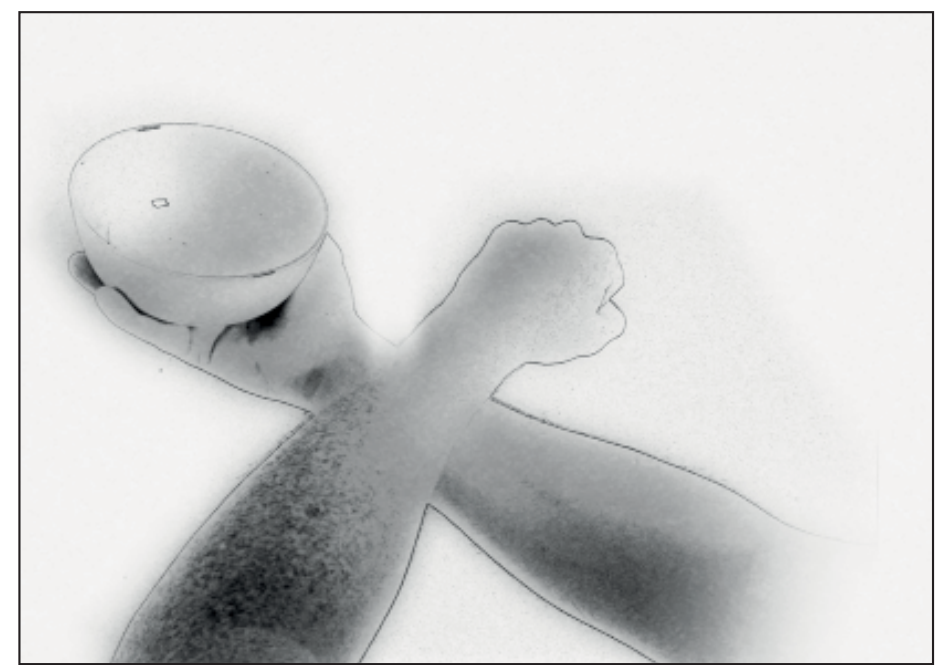

Figure 4 : Le geste du buveur dans le palo monte - C Katerina Kerestetzi

Dans un rituel, si l'on peut parfois boire beaucoup, on ne boit jamais comme dans un bar. Et il existe aussi toute une panoplie de récipients pour l'alcool qui participe à marquer cette différence. Par exemple, on ne boit jamais dans un verre classique mais dans une jícara, une petite coupe constituée d'une demi-calebasse ou d'une demie noixde-coco évidée. Certains servent le rhum dans la corne d'un bouc ou d'un bœuf, nue ou décorée de perles. Et pour accentuer davantage la différence entre alcool profane et alcool rituel, on ne parle pas de rhum ou de vin sec mais on emploie les termes kikongo de « malafo » et de " malafo ensese », respectivement.

Si les morts ont d'étranges manières de boire, ils affectionnent aussi les boissons insolites. A l'eau-de-vie et au rhum pur, les nfumbis préfèrent un cocktail, la chamba, la boisson rituelle du palo monte par excellence. Il s'agit d'un breuvage fait maison, constitué d'eau-de-vie, de piments, d'ail, de poudre à fusil et parfois d'ossements humains pulvérisés. Considérée comme très forte pour les vivants, elle constitue la boisson de prédilection des nfumbis. S'il s'agit de la seule boisson de couleur trouble que l'on consomme dans le culte, c'est parce qu'elle est fabriquée de la main même du palero qui dirige le groupe initiatique et parce qu'elle est sédentaire ; ne quittant jamais l'enceinte du sanctuaire, elle ne risque pas l'empoisonnement.

La chamba est un artefact rituel à part entière qui rend fier son fabricant; les paleros vantent souvent la puissance de leur potion et n'hésitent pas à la tester sur chaque nouveau client ou visiteur qui se présente à leur temple - ceci d'ailleurs, plus dans le but de créer une situation amusante que pour des motifs religieux ou spirituels. On scrute alors chacune de leurs réactions et on les commente, on rit, on se moque de leurs expressions faciales, de leur quinte de toux ou de leurs yeux soudainement injectés de sang. C'est un moment sympathique et décontracté mais pas pour autant vide symboliquement. Au contraire, il suggère de manière éloquente que les paleros et leurs morts, qui boivent de la chamba sans sourciller, sont faits d'une autre étoffe que celle des simples mortels. Du point de vue de celui qui la goûte, cette boisson constitue presque une épreuve rituelle : s'il arrive à contrôler ses effets, c'est qu'il partage l'éthos viril des paleros. Et ce n'est pas un hasard si les pratiquants doivent ingérer la chamba juste après avoir subi la séquence initiatique qui les fait accéder au statut d'initié. On dit alors que si l'aspirant est bienveillant et qu'il est fait pour le palo monte, il supportera 
la potion ; si c'est un ennemi ou une personne arrivée au culte par erreur, elle la vomira.

La chamba est magique en quelque sorte. On la donne aux ensorcelés pour conjurer le mal qui leur est fait. Comme celui-ci résulte souvent de l'ingestion d'un philtre magique, il se loge dans l'estomac. La chamba sait l'extraire. Mais elle agit aussi comme un remède contre les maux d'estomac banals. Pour un médecin qui a prêté le serment d'Hippocrate, la chamba, qui contient des ossements et de la poudre à fusil a tout d'une hérésie mais, pour le palero, l'intensité de son effet est synonyme de catharsis.

La boisson palera participe à la dramaturgie rituelle à plusieurs titres. On peut l'employer pour tester l'authenticité d'une possession par un nfumbi. Par exemple, on peut la vaporiser dans l'air, à la manière d'un gaz lacrymogène et vérifier que le médium qui est possédé résiste à la sensation d'étouffement et d'irritation des yeux qu'elle provoque invariablement chez tous les autres participants. Dans le palo monte, les possessions par les nfumbis sont particulièrement violentes car elles font accomplir à leur médium des actes impossibles en temps normal qui démontrent une résistance physique hors norme - manger du verre, éteindre des cigares incandescents sur sa peau, avaler les intestins des animaux sacrifiés, etc. Ce n'est pas étonnant si la chamba sert à accréditer une possession rituelle, surtout si le médium est une femme. La consommation des spiritueux par les femmes a beau être décomplexée à Cuba, celles-ci conservent tout de même la réputation d'être moins portées sur l'alcool que les hommes. Or, quand une femme est possédée par un esprit mâle, il est fréquent qu'elle demande à boire de faramineuses quantités de rhum, situation qui marque les esprits des autres initiés qui ne reconnaissant plus leur coreligionnaire. Par exemple, au cours d'un rituel de montage d'une nganga, l'esprit qu'on allait introduire dans le chaudron a possédé un médium femme, connue pour ne jamais boire d'alcool en temps normal. Quand les participants l'ont vue saisir la chamba, ils n'ont pu s'empêcher d'exprimer leur étonnement, par des commentaires : «Mon Dieu ! Regardez moi ça ! ( Ave María, mira eso !). Et une fois sorti de sa transe, le médium s'est montré profondément choqué d'apprendre ses aventures alcoolisées. Et comme cela arrive souvent, il s'est s'exclamé : «Oh, non ! Moi, je ne bois pas d'alcool ». Pour les paleros, plus l'écart entre la personnalité du médium et celle du nfumbi est manifeste, plus il accrédite la véracité d'une possession par les morts.

L'alcool n'est pas fait uniquement pour être bu. Il s'agit d'une substance aux propriétés et affordances multiples. Dans l'espace d'un rituel ou d'une consultation, il est particulièrement apprécié pour sa capacité à produire des flammes et pour la vélocité de sa combustion. Il faut noter que les rites paleros plaident pour le spectaculaire et l'étonnant et doivent marquer les esprits. Ces pratiquants ont par exemple développé une grande maîtrise des gestes et des substances pyrotechniques - la poudre à canon, les allumettes et l'alcool pur font d'ailleurs souvent partie des éléments de base d'un lieu de culte palero, prêts à tout moment à être enflammés pour brûler les esprits malins et les mauvaises influences. Les méthodes abondent. Chez la vieille palera Marelis par exemple, on trace sur le sol du sanctuaire un signe magique, une firma (voir Bonhomme et Kerestetzi 2015), on y verse une large quantité d'alcool à brûler puis on l'enflamme à l'aide d'une bougie. Tous ceux qui sont présents dans la pièce se tiennent par la main et forment un cercle autour du feu, puis ils effectuent une ronde dans le sens des aiguilles d'une montre. Le déplacement d'air, induit par le mouvement circulaire des participants, fait s'élever les flammes pendant quelques secondes, ce qui leur donne la 
forme d'une colonne de feu d'un mètre cinquante de haut, phénomène qui ne manque pas d'impressionner celui qui y assiste pour la première fois (Figure 5).

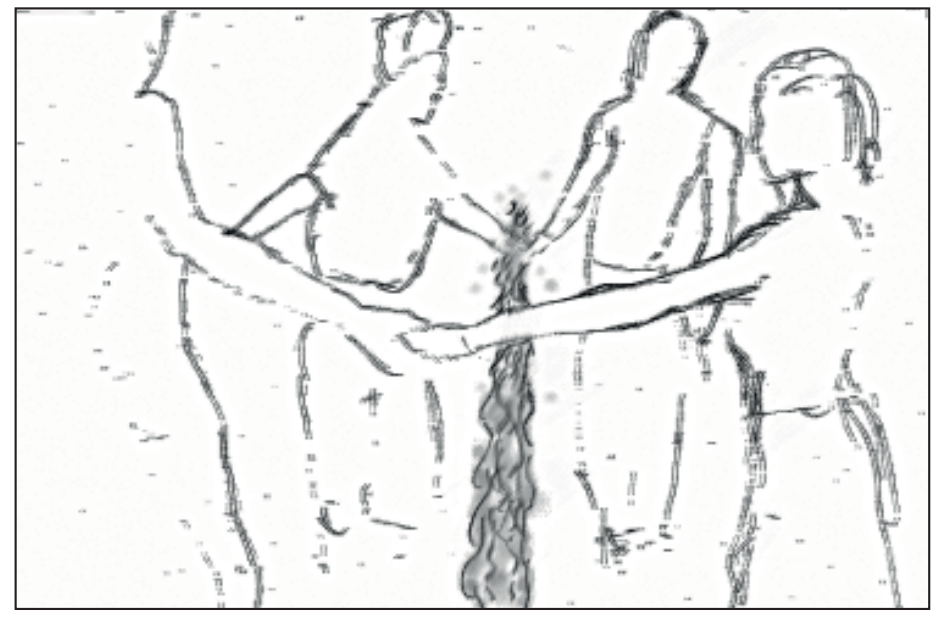

Figure 5 : Brûler le mal - (C) Katerina Kerestetzi

Chez Pedro Tomás, un autre chef rituel, on conjure le mal en crachant du feu. Le patient est debout avec les bras ouverts en forme de croix et les jambes écartées. Le palero prend une grosse quantité d'alcool pur dans la bouche et en tenant une allumette allumée devant ses lèvres, il projette un jet de flammes à différents endroits du corps de son patient: sous le bras droit, sous le bras gauche, entre les jambes, au-dessus de la tête - les endroits symbolisant les quatre points cardinaux, l'acte signifie que le patient sera protégé du mal quelle que soit la direction d'où il vient.

L'imagination technique des pratiquants est-elle aussi souvent mobilisée. Pour produire des flammes, certains pratiquants fabriquent eux-mêmes des engins pyrotechniques, en modifiant certains objets du quotidien (voir Kerestetzi 2015). A l'instar de ce palero qui utilise une carafe de terre cuite dotée de deux orifices, dans laquelle il introduit de l'alcool à brûler (Figure 6). Après avoir secoué le récipient, il introduit une mèche de papier dans l'un des orifices et l'allume. Une gerbe de feu sort alors violemment de l'autre orifice, en émettant un son strident, similaire à un cri. La flamme produite est porteuse de messages et de sens : sa taille et l'intensité du son émis permettent au palero d'interpréter le degré de danger auquel est confronté un client mais aussi de vérifier le résultat d'une conjuration. Si la flamme est très haute, c'est que l'esprit malin qui hantait le client est irrévocablement anéanti.

Si l'alcool est un désinfectant naturel, dans le palo monte il est vu comme un purificateur spirituel. Chez certains paleros, avant de sacrifier un coq ou un bouc, on asperge l'animal d'une large rasade de rhum afin de le purifier des mauvaises influences et de le préparer pour le sacrifice. Ou encore, une fois le « meurtre » rituel accompli, le pratiquant peut laver ses couteaux sacrificiels à l'eau-de-vie et à la fumée de tabac afin d'annihiler leurs vibrations meurtrières.

L'alcool est également utilisé dans les «bains spirituels ». Dans le palo monte, comme dans tous les cultes afro-cubains, les nettoyages rituels (limpiezas) sont monnaie courante et ont cours dans la plupart des rituels. On peut laver une personne en frottant son corps avec un cigare, un poulet vivant, un œuf... Mais quand on veut charger davantage l'action de signification, notamment lors des initiations qui visent 
non seulement à débarrasser l'aspirant de ses maux mais aussi de tout son passé et sa vie de profane, on confectionne un mélange liquide constitué d'eau, d'herbes médicinales, de branches d'arbres (palos) réduits en poudre, de la fumée d'un cigare, de l'eau de coco et de l'eau-de-vie. On lave ensuite le patient en versant ce cocktail sur sa tête et en le frottant avec les herbes. Le frottement de l'alcool irrite la peau et stimule l'odorat, contribuant ainsi pour une petite part au changement d'état ressenti par le patient.

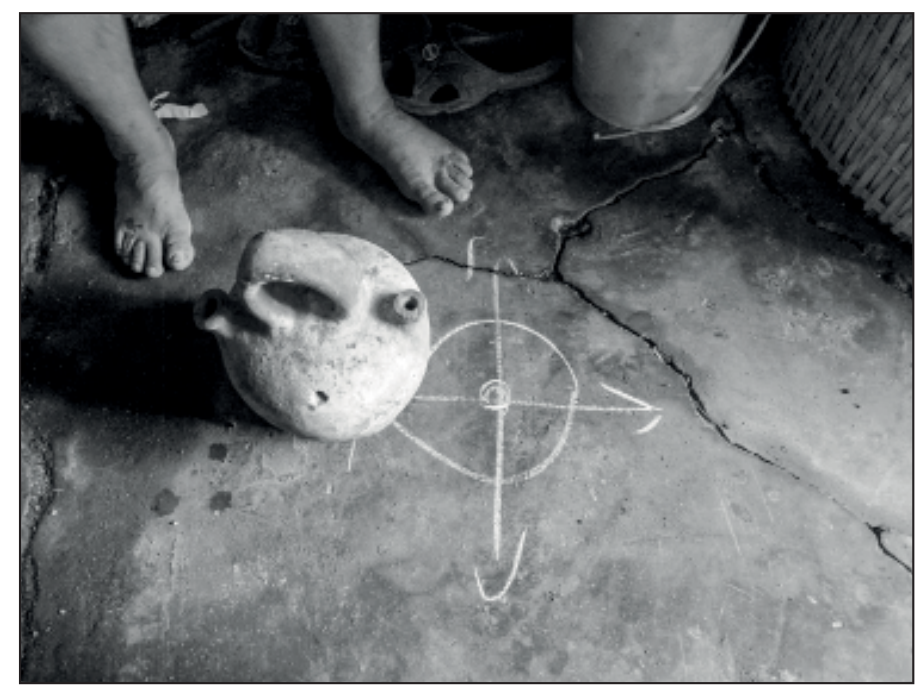

Figure 6 : Engin pyrotechnique posé sur un signe magique - (C) Katerina Kerestetzi

C'est en ce sens qu'agit le parfum utilisé dans le cadre des consultations. Comme l'immense majorité des parfums sont dits « alcooliques », c'est-à-dire qu'ils utilisent l'alcool éthylique comme support de la substance odorante, j'inclus leur mention dans cet article, même si les paleros n'ont probablement pas cela en tête lorsqu'ils les mobilisent. A l'instar de cette vieille pratiquante qui emploie le parfum à chaque purification et conjuration. Par exemple, lorsqu'elle veut vérifier l'efficacité d'une purification, elle demande à ses patients de se tenir debout, les bras en croix, une bougie allumée dans chaque main. Puis, elle vaporise les flammes des chandelles avec un flacon de parfum jusqu'à ce qu'elles s'éteignent. Si elles disparaissent rapidement, c'est que le mort antagoniste a cessé de perturber la personne ; si elles résistent, c'est qu'il faut poursuivre l'exorcisme et employer de plus gros moyens. La combinaison de l'odeur de parfum avec l'attention portée à la flamme, la posture debout et l'effort de garder les bras tendus à l'horizontale, crée un ensemble d'effets sensoriels inhabituels qui, s'ils ne favorisent pas forcément la guérison, induisent, tout au moins, chez le patient le sentiment qu'un changement s'est produit lors de ce rituel (Figure 7). Cet exemple, tout comme le précédent, nous révèlent également que les changements d'états psychologiques produits par l'alcool ne sont pas uniquement fonction de sa capacité à générer l'ivresse par métabolisation mais résultent aussi de sa capacité à exciter les sens, en particulier celui du toucher et de l'odorat. 


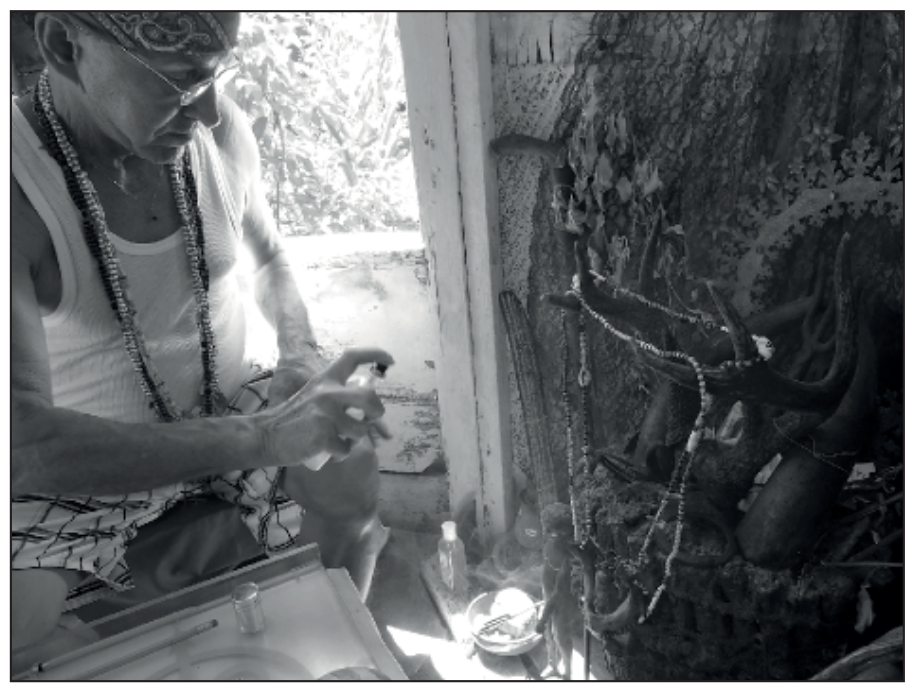

Figure 7 : Palero qui parfume sa ndanga - (C) Katerina Kerestetzi, Cienfuegos 2012

Autre propriété originale de l'alcool plébiscitée par les paleros : celle d'isolant. A chaque fois que l'on opère une incision initiatique sur le corps d'un aspirant, on verse de l'alcool pour la sceller et éviter une infection par le mal. Dans le palo monte, on dit que le mal s'introduit dans le corps par les incisions cutanées. Ainsi, quand on décapite un coq, on veille aussitôt à verser sur sa gorge sanguinolente de l'eau-de-vie ou du rhum afin que sa carcasse ne soit pas infectée par l'intrusion d'un esprit maléfique qui la convoite. On chante alors : "Malafo maputo que bueno es, malafo nganga que bueno es » («Que c'est bon l'eau-de-vie, l'eau-de-vie de la nganga, que c'est bon !»).

Autre dimension intéressante, la possibilité de cumuler les couches d' " isolants » afin de renforcer leurs effets. Ainsi, lorsque la gorge fraîchement tranchée de l'animal vient d'être mouillée de rhum, on vient y rajouter quelques mesures de vin blanc sec, opération que l'on nomme « couvrir » (cubrir). On doit alors chanter : " Malafo ensese que bueno es, que bueno es " (Qu'il est bon le vin sec, qu'il est bon), avant d'enduire le cou sanguinolent de miel, le malafo Chola, c'est-à-dire le « rhum de Mama Chola », afin d'apporter de la douceur à l'acte meurtrier. Mama Chola, ou Ochún dans la santería (le panthéon palero est assimilé à la fois au panthéon santero et aux saints catholiques), la divinité des eaux douces, est l'Eros du palo monte, un cupidon qui instille l'amour dans le cœur des humains, en leur faisant goûter son miel et sa cannelle.

\section{Alcool et style rituel}

Le palo monte est caractérisé par l'extrême variabilité de ses pratiques, à tel point que l'on peut affirmer qu'il existe autant de manières d'accomplir le culte que de groupes initiatiques (Kerestetzi 2016). Conséquemment, la manière d'employer les alcools varie considérablement d'un groupe à l'autre, contribuant ainsi pour une petite partie à les différentier les uns des autres.

Nous avons déjà évoqué l'ambiguïté morale qui caractérise la consommation d'alcool en contexte profane. A Cuba, on le sait déjà, la consommation de rhum et de bière est généralisée et ne pose en tant que telle aucun problème. En revanche, l'ivresse suscite clairement la répugnance, la peur parfois. Parmi les religieux afro-cubains, les paleros sont ceux qui sont considérés comme les plus enclins à l'alcoolisme et à la 
transgression. Les santeros et les babalaos ne manquent d'ailleurs jamais une occasion d'évoquer la nature immorale et déviante des pratiquants du palo monte. Ils peuvent conseiller à leurs clients de ne jamais assister à un rituel palero car là, des hommes ivres règlent leurs comptes à grand coup de couteau et entrent dans des états extatiques qui peuvent mettre en danger leur entourage. Il faut dire qu'il s'agit d'un stéréotype qui est corroboré par les paleros eux-mêmes car il met en scène un éthos viril, explosif et guerroyant qui répugne autant qu'il fascine. En revanche, lorsqu'on interroge les pratiquants sur ce type de comportement, on s'aperçoit que cela n'arrive jamais chez eux mais uniquement chez les autres paleros, les concurrents - ce sont eux qui s'enivrent, qui se bagarrent et qui ne voient dans les rites qu'une bonne occasion d'exprimer leur tempérament agressif. Dans son propre groupe, on dit qu'on boit, non pas parce qu'on le veut, mais parce que le mort le veut - «c'est le mort qui est alcoolique, pas nous »la consommation d'alcool des initiés est ainsi considérée comme un signe de respect. La relation présumée à l'alcool devient ainsi mesure de la moralité du pratiquant. Si au début de cet article nous avons vu que l'ambiguïté morale de la consommation alcoolique était le miroir de l'éthos douteux des morts, ici on voit qu'elle est transposée aux adeptes du culte.

Parfois, lorsque le nfumbi est alcoolique, il exige des participants qu'ils consomment avec lui de grandes quantités d'alcool pendant les rites. Il s'agit d'une demande que l'on ne peut jamais refuser - fêter avec les morts et comme les morts est une obligation rituelle à part entière. Tous les nfumbis boivent et le rhum est présent dans chaque activité palera, on le sait déjà. Or, c'est la quantité qui fait toute la différence. Plus les morts sont alcooliques, plus le rituel devient spectaculaire, festif et convivial. Les cérémonies paleras qui réunissent l'ensemble du groupe initiatique sont des événements rares. Dans certains groupes, ils n'ont lieu qu'une seule fois dans l'année. Ils doivent donc laisser une trace durable dans la mémoire des participants. Créer une ambiance festive, rythmée d'actes extravagants, est une bonne manière de marquer les esprits et il ne faut pas s'étonner si, en général, les cérémonies paleras sont bien arrosées. Certains chefs rituels ont clairement cela à l'esprit lorsqu'ils distribuent abondement le rhum ; les cérémonies sont l'occasion de resserrer les liens entre les membres du groupe et de leur offrir une expérience intense. Mais dans la majorité de cas, la grande consommation d'alcool relève clairement de la croyance : elle est corrélée avec cet alcoolisme du nfumbi que l'on doit obligatoirement satisfaire.

L'une des conséquences de cette ambiance plus relâchée est d'offrir un environnement plus propice à l'expression de possessions violentes. Loin de moi l'idée que c'est la quantité d'alcool bue par un participant qui détermine le degré de violence ou d'extravagance de sa possession, mais j'ai remarqué à de nombreuses reprises que dans les maisons de culte où la consommation d'alcool est plus abondante, les possessions sont plus extraverties. Selon moi, il faut plus y voir l'effet de l'atmosphère plus festive ou permissive qui règne dans les lieux de culte que l'action concrète de l'alcool sur le métabolisme des médiums. Il n'est pas rare de voir ainsi un possédé se jeter de toutes ses forces la tête contre une porte, de se brûler le corps avec des cigares incandescents, de manger à même la bête les intestins des animaux sacrifiés et de se saisir à main nue des serpents. Ce faisant, les médiums respectent les codes de la possession palera, considérée comme très violente ou fougueuse. D'ailleurs, la consommation d'alcool peut devenir elle-même un outil au service de la performance rituelle. On peut ainsi 
voir les possédés boire une pleine bouteille de rhum d'un seul coup, ou essayer de boire par les oreilles. Ces actes extraordinaires feront ensuite partie de l'histoire singulière d'un groupe et ils seront racontés avec fierté à chaque occasion qui s'y prête. Ce motif de l'extravagance, de l'explosivité et de la festivité respecte les attentes que l'on peut avoir d'un rituel palero. Or, la réalité est fort plurielle et il n'est pas rare d'assister à une cérémonie du palo monte où règne calme, douceur et sobriété.

C'est notamment le cas de certaines maisons de culte où le nfumbi n'est pas issu d'un personnage sulfureux mais plutôt d'une « bonne personne ", qui peut parfois même avoir été connue de son vivant par le pratiquant qui en a récupéré l'esprit (je connais l'exemple d'un palero qui a installé l'esprit de sa grand-mère, chrétienne exemplaire, dans sa nganga). Dans ces cas-là, la consommation d'alcool est généralement modérée et on peut quitter une cérémonie aussi sobre que l'on y est entré. Dans ces lieux de culte, les participants boiront quelques gorgées de rhum mais restreindront son usage au maximum afin d'éviter les ivresses qui pourraient vexer les nfumbis abstèmes. On voit ainsi qu'un simple élément de la personnalité du nfumbi, à savoir son rapport supposé à l'alcool, peut avoir des conséquences importantes sur l'ambiance qui règne dans un rituel : euphorique, festive, conviviale, solennelle, sévère, ennuyeuse parfois.

Autre motif d'abstinence alcoolique : la volonté de contrôle du palero sur le déroulement d'une cérémonie. Le cas du chefFelipe Phan est exemplaire. Les cérémonies menées par Felipe - aujourd'hui décédé - étaient étrangement calmes et sobres. Elles ressemblaient plus à des réunions spirituelles, rythmées par les paroles d'un maître qu'aux traditionnelles performances spectaculaires scandées par les tambours. Felipe ne cessait de rappeler que les cérémonies ne sont pas faites pour le plaisir des humains mais pour honorer les morts. Et il imposait certaines règles afin de le faire au mieux. La principale consistait à ne pas consommer d'alcool en dehors des exigences rituelles (interdiction rarement édictée par les chefs de culte), ou si lui-même n'en offrait pas. J'ai assisté un jour chez lui à une cérémonie, de plus de trois heures, au cours de laquelle la jicara ne tourna que quatre fois, ce qui signifie que quatre gorgées par participant. Ici la consommation d'alcool n'a pas de vertu euphorisante. Et ce n'est pas le but ; pour Felipe, dans un rituel, on ne doit pas être joyeux ou extatique mais déférent et concentré. Felipe était un chef de culte charismatique dont la grande popularité - le nombre de ses filleuls s'élevait selon lui à plus de 1000 - reposait en partie sur la morale et la discipline qu'il inspirait. Ses rituels reflétaient cet esprit de respect qui ne laisse pas de place aux gestes maladroits ou à des comportements déplacés, encore moins à des esprits émoustillés. Je n'ai jamais vu autant de retenue et de révérence dans un rituel palero, d'habitude très décontracté et convivial. Des chefs religieux comme Felipe, qui incarnent des idéaux moraux, sont particulièrement recherchés aujourd'hui à Cuba où les charlatans abondent selon l'opinion publique. Ils rendent l'affiliation au palo monte plus légitime en proposant une alternative à la représentation commune du palero - vu comme une personne vénale, avec un fort penchant pour l'alcool, qui ne recule devant aucun crime. Dans tous les cas, les modalités de distribution de l'alcool faisant l'objet d'attentions rituelles font du boire un acte rituel à intégrer dans le répertoire praxique d'un groupe. 


\section{Conclusion}

Pour analyser l'action et l'existence humaine, Albert Piette (2004) invite à adopter le " mode mineur ", une approche qui fait la part belle à ce qui reste dans l'ombre de la réflexion anthropologique et en dehors des carnets de terrain - les détails des actions, les petits gestes involontaires des mains, les mouvements discrets du regard, les moments de distraction, les fluctuations de l'attention, les interactions banales et infimes. Sa démarche est « ego-centrée », en ceci qu'elle concerne essentiellement les aspects phénoménologiques et cognitifs de l'être. Ici, j'ai souhaité adopter cette approche attentive au banal et au détail mais en l'appliquant à la sphère des matières du religieux, à savoir mettre en évidence un mode d'existence des objets rituels mineurs, pour analyser des faits majeurs et collectifs.

Nous avons vu que l'alcool, un produit de consommation commun, était approprié par le palo monte et transformé en un outil rituel à part entière, en source de sensation religieuse et d'information, en moyen de communication, en monnaie spirituelle et en ressort performatif et identitaire. En questionnant un élément en apparence accessoire de la liturgie, nous avons donc abordé des questions aussi importantes que l'organisation des rituels ou la construction d'un éthos religieux. La démarche fut-elle féconde parce que nous avons identifié un élément en réalité incontournable du culte ? Certainement. Et il est évident que tous les objets rituels n'ont pas la même valeur heuristique car ils n'ont pas la même agentivité ni le même rôle dans le façonnement des pratiques. L'étude des trajectoires d'une nganga livrerait, sans doute, plus d'enseignements sur le palo monte qu'une simple boîte d'allumettes par exemple. Pourtant, et c'est là tout l'intérêt de cette démarche pragmatique, il y aurait là encore beaucoup de choses à retirer de l'étude de ces modestes allumettes. Le fait que ce soient elles, et non des briquets, qui sont exclusivement utilisées pour produire le feu, nous conduirait à interroger le caractère systématique d'idéal de la nature dans le palo monte. Nous verrions aussi qu'une allumette peut être effritée pour remplacer la poudre à canon, étêtée puis compactée pour servir d'explosif, qu'elle peut être introduite dans les cigares utilisés dans les purifications... Ses trajectoires et ses usages, comme ceux de n'importe quel objet ou substance figurant dans un sanctuaire, nous révèlerait tout un pan d'une pratique rituelle. Et la comparaison de ces usages de sanctuaire à sanctuaire afficherait la part d'idiosyncrasie de chacun et témoignerait de l'extraordinaire inventivité dont font preuve les paleros. 


\section{Références citées}

Argyriadis, Kali, 1999. La Religión à la Havane. Paris : Archives Contemporaines.

Bonhomme, Julien et Katerina Kerestetzi, 2015. « Les signatures des dieux. Graphismes et action rituelle dans les religions afro-cubaines », Gradhiva, 22, pp. 74-105.

Bonvini, Emilio, 1996. « Mets afro-brésiliens. Cuisine des hommes, nourriture des dieux », Journal des Africanistes, 66 (1-2), pp. 137-165.

Cabrera, Lydia, 1998 [1954], El Monte. La Havane : Universal.

Castellanos Jorge et Isabel Castellanos, 1992. Cultura Afrocubana 3 : las religiones y las lenguas. Miami (FL) : Universal.

DiETLER, Michael, 2006. "Alcohol: Anthropological/Archaeological Perspectives", The Annual Review of Anthropology, 35, pp. 229-249.

EsPÍRITO SANTO, Diana, 2015. Developing the dead mediumship and selfhood in Cuban espiritismo. Gainesville (FL) : University Press of Florida.

GefFroy, Céline, 2012. "La gestuelle du buveur dans le contexte festif andin (notes de recherche) 》, Anthropologie et Sociétés, 36 (3), pp. 77-94.

GIBSON, James J., 1977. “The Theory of Affordances”, in Robert SHAw et John BrAnSFOrD (eds), Perceiving, acting, and knowing. Toward an ecological psychology, pp. 67-82. New York : Halsted Press Division, Wiley

Heath, Dwight B., 1987. "Anthropology and alcohol studies: current issues", The annual review of anthropology, 16, pp. 99-120

KARDEC, Allan, 2006 [1857]. Le livre des esprits : questions et réponses des esprits sur dieu, l'au-delà et le sens de la vie. Paris : J'ai lu.

Kerestetzi, Katerina, 2013. «Pénétrer le monde des morts (Cuba) : la constitution du sujet initiatique du palo monte (Cuba, Cienfuegos) », Ateliers d'anthropologie, $38<\mathrm{http}$ //ateliers.revues.org/9357>.

KerestetzI, Katerina, 2015. «Alarmes naturelles, alarmes rituelles : perception et détection du danger dans le culte du palo monte (Cuba) », Ethnologie Française, 45 (1), pp. 143-153.

KeRESTETZI, Katerina, 2016. Vivre avec les morts à Cuba : réinvention et transmission religieuse dans le palo monte. Paris : Karthala.

Mandelbaum, David G., 1965. “Alcohol and Culture”, Current Anthropology, 6 (3), pp. 281-288, $289-293$.

OrTIZ, Fernando, 2001 [1906]. Los negros brujos: apuntes para un estudio de etnología criminal. La Havane : Ciencias Sociales.

Palmié, Stephan, 2002. Wizards \& Scientists: explorations in Afro-Cuban modernity \& tradition. DurhamLondres : Duke University Press.

PietTe, Albert, 2004. «L'anthropologie existentiale : présence, coprésence et leurs détails », Antrocomn, 4 (2), pp. 99-103.

Pitrou, Perig, 2012. "Figuration des processus vitaux et co-activité dans la Sierra Mixe de Oaxaca (Mexique) », L'Homme, 202, pp. 77-112.

Rougeon, Marina et Jorge Santiago, 2017. Manger avec les esprits. Offrandes, partages et soins dans l'umbanda au Brésil et au Portugal. Paris : Éditions des Archives Contemporaines.

StÉPANOFF, Charles, 2014. «Technologies cognitives du voyage chamanique. Cas iakoutes », in Carlos FAUSTO et Carlo SEVERI (éds), Image rituelle : agentivité et mémoire, pp. 112-146. Paris : L’Herne (Cahiers d'anthropologie sociale, 10). 